\title{
A Rare Case of Morbidly Adherent Placenta in a Primigravida
}

\author{
${ }^{1}$ Hemlata Kuhite, ${ }^{2}$ Sharayu Mirji, ${ }^{3}$ Sangeeta Shingatgeri, ${ }^{4}$ Ganesh Shinde
}

\begin{abstract}
Aim: To report the occurrence of an adherent placenta in the absence of prior risk factors and discuss the various management options especially conservative management.

Background: Morbidly adherent placenta (MAP) refers to any placental implantation with abnormally firm adherence to myometrium. Morbid adherence of placenta has evolved into one of the most serious problems in obstetrics. The incidence has increased tenfold in the past 50 years due to the increasing number of cesarean sections and has reached seemingly epidemic proportions. The American College of Obstetricians and Gynaecologists cites the incidence to be as high as 1 in 533 deliveries.
\end{abstract}

Case report: We report a case of the placenta increta in a primigravida successfully managed by a conservative method with injection methotrexate followed by uterine artery embolization.

Conclusion: Selected cases of the morbidly adherent placenta can be successfully managed conservatively. With proper selection of cases and adequate monitoring, modern conservative techniques have made preservation of fertility possible.

Clinical significance: Only four cases of the adherent placenta in primigravida without any risk factors have been reported in the literature. Fertility preservation is a major concern in the management of these patients.

Keywords: Adherent placenta, Conservative management, Primigravida, Uterine artery embolization.

How to cite this article: Kuhite H, Mirji S, Shingatgeri S, Shinde G. A Rare Case of Morbidly Adherent Placenta in a Primigravida. World J Anemia 2018;2(2):74-77.

Source of support: Nil

Conflict of interest: None

\section{BACKGROUND}

Morbidly adherent placenta (MAP) refers to any placental implantation with abnormally firm adherence to myometrium. The American College of Obstetricians and

\footnotetext{
${ }^{1}$ Assistant Professor, ${ }^{2}$ Consultant, ${ }^{3}$ Assistant Honorary, ${ }^{4}$ Professor and Head

${ }^{1-4}$ Department of Obstetrics and Gynecology, HBT Medical College and Dr RN Cooper Municipal General Hospital, Mumbai, Maharashtra, India

Corresponding Author: Sharayu Mirji, Consultant, Department of Obstetrics and Gynecology, HBT Medical College and Dr RN Cooper Municipal General Hospital, Mumbai, Maharashtra, India, e-mail: sharayumirji@gmail.com
}

Gynaecologists cites the incidence to be as high as 1 in 533 deliveries from the period from 1982 to $2002 .{ }^{1}$

The incidence of them MAP has increased 10-fold in the past 50 years owing to the rising cesarean section rates worldwide. ${ }^{2}$ It is now an important cause of interact postpartum hemorrhage, maternal morbidity, and mortality and is a leading cause of peripartum hysterectomy. In cases of placenta percreta, the placenta may invade into surrounding structures. Berg et al. reported that around $8 \%$ of maternal deaths due to hemorrhage were caused by the morbidly adherent placenta. ${ }^{3}$ The various risk factors of MAP include prior cesarean section, placenta previa, prior hysterotomy, uterine curettage, myomectomy, submucous fibroids, Ashermans syndrome, advanced maternal age, grand multiparity, smoking, chronic hypertension. ${ }^{2}$

We present a rare case in which MAP was encountered in a primigravida with no previous uterine surgeries and no other risk factors. The patient was managed conservatively with injection methotrexate and uterine artery embolization thus preserving her fertility.

\section{CASE DESCRIPTION}

A 25-year-old Asian female, primigravida, conceived spontaneously, 29 weeks of gestation by date, reported to our obstetric casualty with chief complaints of leaking per vaginum for four days. No other complaints. No significant past medical or surgical history.

On examination, vital parameters were stable. The leak was found to be present, and membranes were absent. Liquor was clear. She was admitted because of preterm premature rupture of membranes. After hospitalization, routine investigations including C-reactive protein (CRP) were sent. Ultrasonography was suggestive of single live intrauterine gestation of $27+2$ weeks, cephalic presentation with severe oligohydramnios, amniotic fluid index $0-1$ $\mathrm{cm}$. Estimated birth weight $1.033 \mathrm{~kg}$. Placenta was fundal.

Parenteral antibiotics and steroids were administered. In spite of no fever, infection was suspected, as there was a rise in WBC count from 13,600-24,900 and doubling of the CRP titers from 1:16-1:24. Because of the risk of developing chorioamnionitis, labor was induced with intracervical dinoprostone gel. Induction to delivery time was 20 hours. She delivered a preterm male child of $1.095 \mathrm{~kg}$, with an Apgar score of 9/10 and was shifted to a neonatal intensive care unit. 
Despite uterine massage and uterotonics and watch for one hour, placenta failed to deliver. Cervical os was open, but there was no active vaginal bleeding. Manual removal of placenta was attempted in the operation theatre under general anesthesia, the placenta was found to be fundal, and no cleavage plane could be made out between the placenta and the uterus. The patient was hemodynamically stable; hence the procedure was abandoned. A diagnosis of adherent placenta was made.

Ultrasound of the pelvis with doppler was done. It showed evidence of retained placenta (180 cc volume) and thinned myometrium at the placental site suggestive of myometrial invasion-placenta accreta. Pelvis magnetic resonance imaging (MRI) with contrast done. It showed evidence of placenta accreta (severe thinning of overlying myometrium with the irregular placental-myometrial interface). Figures 1 and 2 show the MRI plates depicting placenta increta-T2 weighted images in coronal and axial section, respectively.

As the patient was vitally stable and not bleeding actively, the decision of conservative line of management was taken after detailed counseling. The patient was monitored regularly on the basis of her vital parameters and laboratory investigations, hemoglobin $(\mathrm{Hb})$ and serum levels of $\beta$-hCG (beta subunit of human chorionic gonadotropin), were done on alternate days and pelvic ultrasound for placental volume repeated after two days.

Injection methotrexate $1 \mathrm{mg} / \mathrm{kg}$ intramuscular was given on a postpartum day 1 . There was a rise in serum $\beta$-hCG levels from 18,600 on day 1 to 26,100 on day 5 . The second dose of methotrexate was given on day 5 . Because of falling $\mathrm{Hb}$, two units of blood were transfused. Because of low $\mathrm{Hb}$, rising $\beta$-hCG titers and insignificant decrease in the placental volume, decision uterine artery embolization (UAE) was taken.

The procedure was done on a postpartum day 7 . Twenty-four hours later, sonography revealed placenta

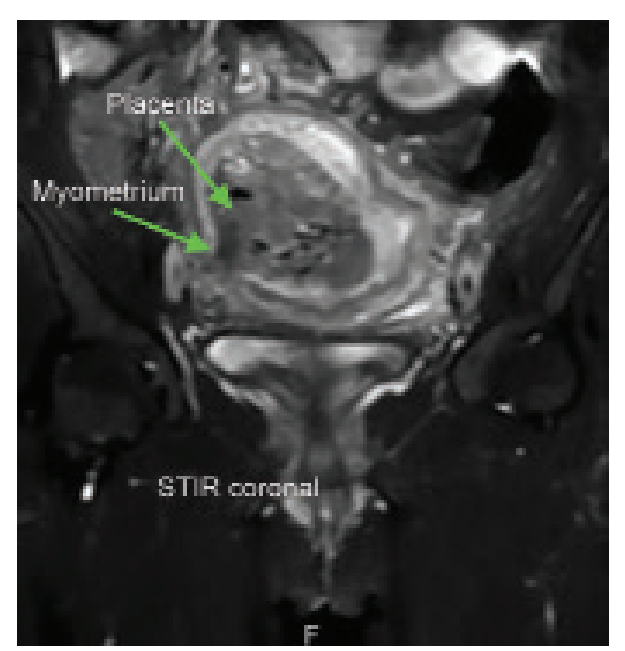

Fig. 1: MRI pelvis in coronal section showing placenta increta (volume $110 \mathrm{cc}$ ) showing post-embolization changes with no significant vascularity. Serial $\beta$-hCG and USG revealed findings suggestive of significant shrinkage of the placenta (day $21 \beta-h C G<2$, placental volume $10 \mathrm{cc}$ ). Table 1 denotes the serial values of serum levels of $\beta$-hCG and placental volume after the embolization procedure.

She continued to have vaginal discharge for 10-12 days following UAE. Discharged on day 14 with weekly follow-up visits. She resumed menses one month later. She is following up with us for 6 months now and has regular menstrual cycles. We will have to wait and see how her future obstetric career shapes up.

\section{DISCUSSION}

Morbidly adherent placenta (MAP) refers to any placental implantation with abnormally firm adherence to myometrium. MAP is a potentially life-threatening condition.

Any prior uterine procedure is a risk factor for morbid adherence of placenta. ${ }^{2}$ There has been a marked increase in the incidence of MAP in recent years mainly attributed to the increased rate of cesarean sections. ${ }^{2}$

It is extremely rare for morbidly adherent placenta to occur in the absence of any risk factors.

Our index case, a primigravida with none of the above-mentioned risk factors (no prior uterine procedure), gave us a shocking revelation during failed manual removal of the placenta in operation theatre. It is difficult

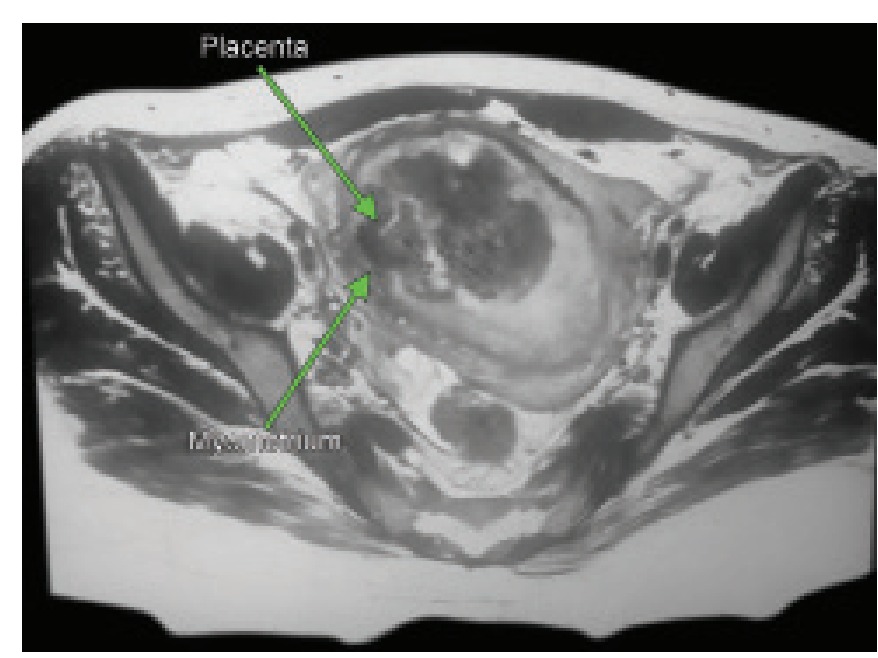

Fig. 2: MRI in axial section showing placenta invading the myometrium

Table 1: Serial values of serum levels of $\beta$-hCG and placental volume following embolization procedure.

\begin{tabular}{lll}
\hline Post-UAE & Sr $\beta$-hCG (IU) & Placental volume (cC) \\
\hline Day 2 & 1776 & 110 \\
Day 9 & 52.75 & 85 \\
Day 14 & 10.94 & 70
\end{tabular}


to diagnose such cases antenatally, given the absence of any antecedent history.

A review of the literature revealed only four such cases of a primigravida with morbidly adherent placenta.

A case of placenta percreta in a primigravida has been reported by Ansar et al., which presented with ruptured uterus at 17 weeks of gestation. It was managed surgically, but uterus could be conserved. ${ }^{4}$

A case of placenta increta in a primigravida was reported by Arnadottir et al. that resulted in the delivery of a healthy infant with successful conservative management with methotrexate. ${ }^{5}$

Kinoshita et al. from Japan, reported a case of spontaneous rupture of the uterus due to placenta percreta in a primigravida. ${ }^{6}$

Another case of placenta percreta in a primigravida with no risk factors was reported by Rajkumar et al., a cesarean hysterectomy was done in view massive hemorrhage. $^{7}$

A high index of suspicion is needed for accurate antenatal diagnosis. Today, antenatal diagnosis is possible with new modern techniques available. The role of ultrasound combined with color Doppler provides an accurate record of the size of the placental mass, the depth of myometrial invasion, the plane of cleavage and blood flow within this mass. MRI findings like uterine bulging, heterogenous placenta, placental bands provide a more accurate assessment of placental invasion. ${ }^{8}$

Antenatal workup helps plan the management. The anticipation and planning of surgery for suspected placenta accreta enable logical and timely decisions to be made without the element of surprise and often without the urgency of massive hemorrhage. ${ }^{9}$

However, very rarely morbidly adherent placenta may be encountered unexpectedly in primigravidae and those without any risk factors.

Also, our patient delivered an extremely premature and low birth weight baby with low chances of survival. Hence, fertility preservation was a primary concern in her management. Fortunately, conservative management could be contemplated as she was hemodynamically stable.

A multidisciplinary approach involving the obstetrician, neonatologist, surgeon, interventional radiologist, pathologist, transfusion medicine specialist is important in managing these patients to reduce morbidity and mortality associated with MAP. ${ }^{10}$

The standard management for confirmed cases of morbidly adherent placenta has been cesarean hysterectomy as described by Fox in $1972 .{ }^{11}$ An alternate approach of conservative rather than extirpative treatment was first described by Arulkumaran et al. in $1986 .{ }^{12}$
In a study conducted by Kayem et al. a comparison was made between the outcomes of conservative and extirpative management of placenta accreta. They concluded that leaving the placenta in situ is a safe alternative to hysterectomy. ${ }^{13}$

Conservative management may be attempted in carefully selected cases, with hemodynamic stability, normal coagulation status, desire for fertility preservation. Thorough prior counseling regarding risks involved in conservative management is mandatory. There are multiple options available including methotrexate administration, uterine artery ligation, internal iliac artery ligation, uterine artery embolization and radiofrequency ablation to name a few. ${ }^{14}$

The need for a close follow-up, repeated investigations, the risk of postpartum hemorrhage, infection, treatment failure, and thereby the probability of hysterectomy cannot be overemphasized.

Continued uterotonic support is generally recommended to keep the uterus well contracted and reduce the risk of postpartum hemorrhage. Methotrexate is a folate antagonist, acts primarily against rapidly dividing cells, and therefore is effective against dividing trophoblast. Tong et al. pioneered the conservative method by administering systemic methotrexate. The outcome varies widely ranging from expulsion at 7 days to progressive resorption in roughly 6 months. ${ }^{15}$ To date, there are no standard dosing regimens or protocols. Some studies have questioned the benefit of methotrexate and noted little benefit in enhancing placental resorption. ${ }^{16}$ After delivery, the trophoblasts are no longer dividing, thereby rendering methotrexate ineffective. ${ }^{2}$ This was probably the reason why methotrexate was not effective in our case.

Bilateral UAE has been used to reduce blood loss and avert surgery. Using local anesthesia, under fluoroscopic guidance, a 4 or 5 French or a microcatheter is inserted percutaneously into the femoral artery and manipulated into the uterine artery via the anterior division of internal iliac artery. Once a stable position is obtained, the embolic agent is injected (polyvinyl alcohol, gel foam, polyacrylamide).

In a study by Perez-Delboy et al., the success rate of pelvic artery embolization in combination with conservative management has been reported as $85-95 \% .{ }^{17}$

It acts by reducing the uterine blood flow thereby causing necrosis and infarction of the placenta ultimately leading to spontaneous expulsion or resorption of the retained placenta.

UAE has an estimated complication rate of $11 \%$. Complications include allergy to contrast medium, hematomas at the puncture site, postprocedural pain, fever, infection, amenorrhea, uterine scarring, and long 
term sequelae like endometrial atrophy, premature menopause. ${ }^{18}$ The impact of UAE on ovarian function warrants further research. ${ }^{19}$

Chauleur et al. showed that return of normal menses and subsequent fertility was not impaired in patients who underwent UAE for post-partum hemorrhage. ${ }^{20}$

A study by Descargues et al. infers that women who undergo arterial embolization for adherent placenta have a return of normal menses and no adverse effects on fertility. However, it is difficult to conclude because of the small number of patients. ${ }^{21}$

\section{CONCLUSION}

In spite of the absence of risk factors, morbidly adherent placenta can be encountered and as seen in the above case can be managed conservatively thereby preserving the uterine function and fertility.

The efficacy of uterine artery embolization as a therapeutic modality for the conservative management of adherent placenta needs further case studying.

Morbidly adherent placenta in a primigravida presents a tremendous challenge to the obstetrician, requiring a more sensitive approach with fertility preservation as a primary concern.

\section{CLINICAL SIGNIFICANCE}

It is extremely rare for morbidly adherent placenta to occur in a patient with no prior risk factors. Fertility preservation is a primary concern in these cases, and hence the conservative approach needs to be studied and evaluated more.

\section{REFERENCES}

1. Wu S, Kocherginsky M, Hibbard JU. Abnormal placenta-: twenty-year analysis. Am J Obstet Gynecol 2005;192:1458-1461.

2. ACOG committee opinion. Placenta accreta. Number 266, January 2002. American College of Obstetricians and Gynecologists. Int J Gynaecol Obstet 2002 Apr;77(1):77-78.

3. Berg CJ, Callaghan WM, Syverson C, Henderson Z. Pregnancyrelated mortality in the United States, 1998 to 2005. Obstetrics and Gynecology 2010 Dec 1;116(6):1302-1309.

4. Ansar A, Rauf N, Bano K, Liaquat N. Spontaneous rupture of primigravid uterus due to morbidly adherent placenta. J Coll Physicians Surg Pak 2009 Nov;19;732-733.

5. Arnadottir BT, Hardardóttir H, Marvinsdóttir B. Case report seventeen year old primipara with placenta increta treated with methotrexate. Laeknabladid 2008;94(7-8):549-552.
6. Kinoshita T, Ogawa K, Yasumizu T, Kato J. Spontaneous rupture of the uterus due to placenta percreta at 25-weeks of gestation: a case report. Journal of Obstetrics and Gynaecology Research 1996;22(2):125-128.

7. Rajkumar B, Kumar N, Sowmya S. Placenta percreta in primigravida, an unsuspected situation. Int Jour of Reprod, Contracept, Obs \& Gyn [Internet] 2014;3(1):239-241.

8. Berkley EM, Abuhamad AZ. Prenatal Diagnosis of Placenta Accreta. Journal of Ultrasound in Medicine 2013;32(8):13451350.

9. Praevia P. Placenta Praevia Accreta and Vasa Praevia: Diagnosis and Management (Green-top Guideline No. 27). Royal College of Obstetricians and Gynaecologists. 2011.

10. Rajkumar B, Kumar N, Srinivasan S. Placenta percreta in primigravida, an unsuspected situation Int J Reprod Contracept Obstet Gynecol 2014 Mar;3(1):239-241.

11. Fox H. Placenta accreta 1945-1969. Obstet Gynecol Surv 1972; 27:475-479.

12. Arulkumaran S, Ng CS, Ingemarsson I, Ratnam SS. Medical treatment of placenta accreta with methotrexate. Acta Obstet Gynecol Scand 1986;65:285-286.

13. Kayem G, Davy C, Goffinet F, Thomas C, Clément D, Cabrol D. Conservative versus extirpative management in cases of placenta accreta. Obstet Gynecol 2004;104:531-536.

14. Timor-Tritsch. Early placenta accreta and cesarean section scar pregnancy: a review. Am J Obstet Gynecol 2012.

15. Tong SY, Tay KH, Kwek YC. Conservative management of placenta accreta: Review of three cases. Singapore Med J 2008;49:e156-159.

16. Timmermans S, van Hof AC,Duvekot JJ. Conservative management of abnormally invasive placentation. Obstet Gynecol Survey 2007:62:529-539.

17. Perez-Delboy A, Wright JD, Surgical management of placenta accrete: to leave or remove the placenta ? BJOG 2014;121: 163-170.

18. Alanis M, Hurst BS, Marshburn PB, Matthews ML. Conservative management of placenta increta with selective arterial embolization preserves future fertility and results in a favorable outcome in subsequent pregnancies. Fertility and Sterility 2006 Nov 1;86(5):1514-e3.

19. Chrisman HB, Saker MB, Ryu RK, Nemcek Jr AA, Gerbie MV, Milad MP, et al. The impact of uterine fibroid embolization on resumption of menses and ovarian function. Journal of Vascular and Interventional Radiology 2000 Jun 1;11(6): 699-703.

20. Chauleur C, Fanget C, Tourne G, Levy R, Larchez C, Seffert $\mathrm{P}$. Serious primary post-partum hemorrhage, arterial embolization and future fertility: a retrospective study of 46 cases. Hum Reprod 2008;23:1553-1559.

21. Descargues G, Mauger Tinlot F, Douvrin F, Clavier E, Lemoine JPL, Marpeau. Menses, fertility and pregnancy after arterial embolization for the control of postpartum haemorrhage Hum. Reprod 2004;19(2):339-343. 